\title{
A NEW OPTICAL TECHNIQUE FOR MACHINE GUIDANCE
}

\author{
Prof. Barry Gorham \\ Industrial Metrology Research Unit (IMRU), University of East London, \\ United Kingdom. Email: gorham@uel.ac.uk
}

\begin{abstract}
After briefly reviewing earlier developments by IMRU of automatic theodolites that were based on the projection of shaped laser beams, the paper describes a novel design of automatic electronic theodolite that operates entirely as an optical receiver. The theoretical basis for the measurement process is introduced in terms of instrument geometry and in relation to the emissions from a single luminous target. This is followed by a description of two embodiments of the instrument design adapted for reception of luminous emissions from a number of point targets which has application in the process of site vehicle automatic guidance.
\end{abstract}

Keywords: optical instruments; theodolite; surveying; machine guidance.

Since the late seventies, the Industrial Metrology Research Unit (IMRU) of the University of East London has been developing novel instruments for precise and automatic spatial measurements. It developed and then provided a public demonstration of the world's first robotic, surveyors 'Total Station' - LASERFIX, in 1983. The first commercial version appeared some ten years later from Geotronics of Sweden.

In 1989, IMRU designed and produced a working prototype (for British Coal) of the first automatic tunnel guidance system generating 6-D information (position and spatial orientation) of the tunnelling machine in real-time -

LASERGUIDE.

Currently, IMRU is a partner in an international research consortium funded by the European Union Brite-Euram initiative. The project is defined as Computer Integrated Road Construction (CIRC), and is concerned with automation of the road construction process from design office to work-site. Here, the specific remit for IMRU is to develop an automatic 6-D laser dynamic guidance system for road pavers.

A common feature of all of the foregoing geometrical measurement systems is the application of the projection of compound scanning laser beams from an automatic theodolite instrument. The laser theodolite uses a single electronic angle scale to measure automatically in each scanning cycle the full direction vector (both horizontal and vertical directions) to each individual target in its field of view. The targets are either single photocells or glass corner-cube retro-reflecting prisms.
For short range applications, such as in the spatial monitoring of end-effectors of in-situ production robots, the laser scanner can operate at speeds of up to $30 \mathrm{~Hz}$. In surveying and machine guidance applications, a more typical scanning speed is 1 or $2 \mathrm{~Hz}$. Typical precision of direction measurement achieved by this system is $+/-1$ arc second. Range measurements are achieved by sighting to known target arrays.

There are many advantages of this form of automatic laser scanning theodolite over its nearest equivalent, the robotic Total Station.

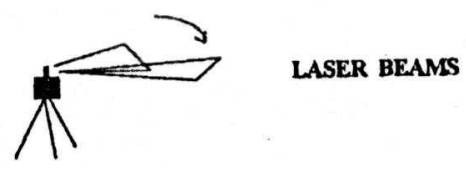

Fig. 1

For one thing, the directions are measured to all of the targets within the $360^{\circ}$ field of the instrument, in less than one second. This allows a computation of $X Y$ and $Z$ position co-ordinates for each target in their array of known relative positions, and accordingly, also its geometrical disposition or orientation in space from the same measurements data.

In contrast, robotic Total Stations can measure $\mathrm{XY}$ and $\mathrm{Z}$ position co-ordinates rapidly to but a single target. Provided the target array is not 
moving, subsequent measures to each target of the array can produce values for the 6-D coordinates of the array itself. The main disadvantages, however, for the robotic Total Stations, are their design complexity and high cost compared with the simple design of a laser scanner instrument.

However, there are two practical limitations to the Laserfix design. The first is a need to correlate the incidence of a scanning laser beam on a distant target with the corresponding and simultaneous reading of the instrument internal angle scale. The second is the need during manufacture to produce two fan-shaped laser beams of geometry known to a high order of accuracy. Accordingly, IMRU has developed a new type of automatic theodolite that offers comparable performance whilst avoiding the previously identified design limitations.

The new design of automatic theodolite contains no laser or any other kind of light source but, in common with traditional optical theodolites, relies only on viewing light emanating from a distant target. Instead of the eye, electronic detection is used and the basic instrument uses only one electronic angle scale. The principle of operation of this design is shown below in Fig. 2.

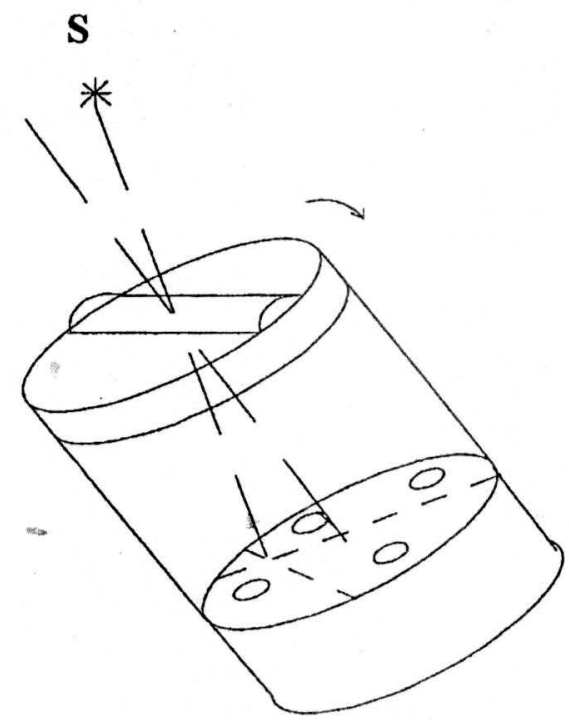

Fig. 2

The essential features of the instrument are contained in a cylindrical housing, rotatably mounted on an internal fixed baseplate. The latter carries a set of photoelectric cells, four in the figure, which are disposed at the corners of a square. There is also provided a thin cylindrical glass lens firmly attached across a diameter at the open end of the cylindrical housing. Further, and internal to the housing, under the baseplate, (not shown in the figure), is an electronic incremental angle encoder disc.

In operation, light from a distant luminous source, such as an LED or laser, enters the cylindrical lens at the front of the theodolite and generates a line image on the baseplate situated in the principal focal plane of the lens. The rotation of the lens, typically between 10 and $30 \mathrm{~Hz}$, is continuously monitored by the angle encoder in the baseplate. Six arc minutes electronic angle markers are time interpolated to produce a resolution of 1 arc second for the instantaneous angle readings latched at the instants that the optical line image passes through a photocell.

Fig.2 shows the line of incidence for a ray of light from a distant source target ' $S$ ' which passes through the centre of rotation of the cylindrical lens and strikes the baseplate at a point displaced from the centre of the photocell array. The latter is indicated by the axis line without notation.

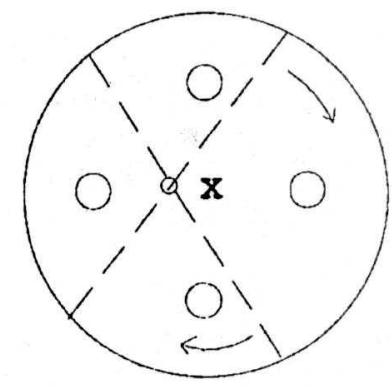

Fig. 3

Fig. 3 shows a plan view of the baseplate and its photocells. Two positions for the rotating line image generated from a single target are shown in the figure and, since the target is assumed to lie in a direction divergent from the instrument axis, the centre of rotation of the line image is offcentre at ' $\mathrm{X}$ '.

It is clear that if the lines joining opposite pairs of photocells define two mutually perpendicular axes, then the linear offsets of the centre of rotation of the line image from these respective axes must correspond to equivalent perpendicular angular offsets from the instrument optical axis. 
Thus by a determination of the position of the centre of optical rotation of the line image across the baseplate, two perpendicular components of spatial direction necessary to define the full direction vector of the distant target may be found. Such a full measure of direction would then be available for each cycle of rotation of the cylindrical housing of the instrument.

Clearly, the angular field of view of the instrument as well as its angular resolution are determined largely by the focal length of the single cylindrical lens, the geometry of the photocell array in the baseplate and the small optical size of the individual photocells. For the latter, small quadrant photoelectric cells seem to offer the best combination of precision and stability. It is also desirable to ensure that the luminous source is modulated at a frequency above $5 \mathrm{MHz}$ in order to reduce the effects of ambient light sources and improve detection.

Further, there is sufficient mechanical inertia to ensure that time interpolation between angle pulses does not reduce precision. There is thus no need to maintain a constancy of rotation of the instrument housing.

Another embodiment of this design uses a single radius cylindrical lens rather than one of a full diameter. This is shown below in Fig. 4.

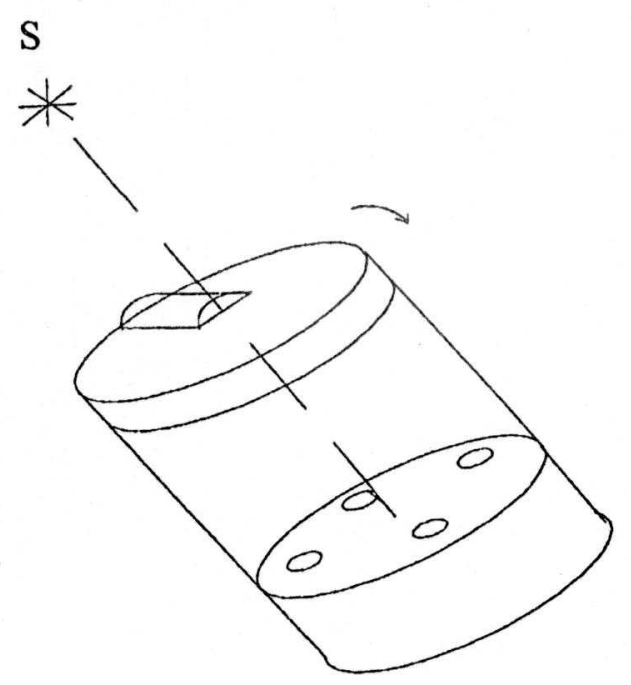

Fig. 4

The arrangement of Fig. 4 produces a rotation of shorter line images about the index point set by angular subtense of the source. The resulting pattern on the baseplate is shown in Fig. 5.

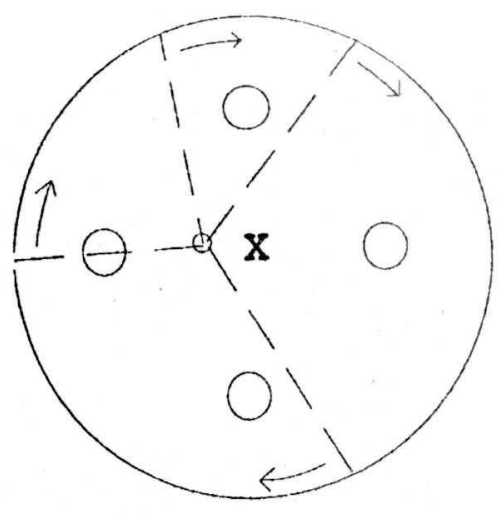

Fig. 5

One advantage of this reduced line image is that any possible ambiguities arising from simultaneous incidence on two photocells by the rotating line image is removed. All signals are now sequential.

A more complete analysis of this method of direction measurement can be made with reference to Fig. 6.

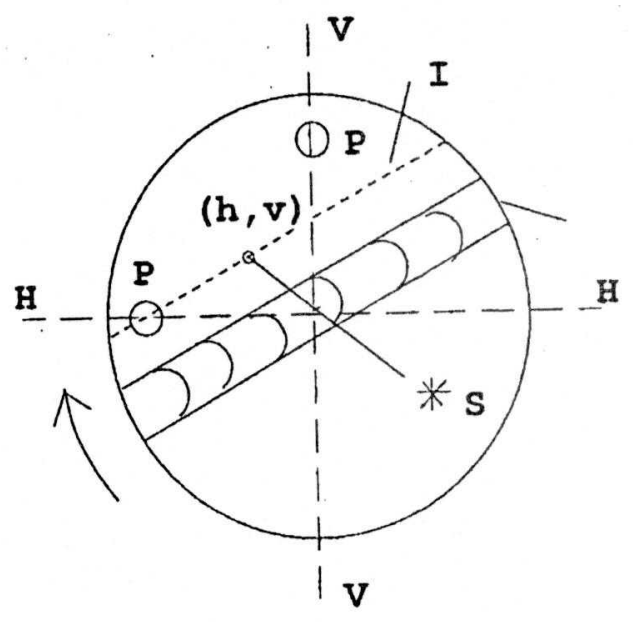

Fig. 6

The offset plan view of the baseplate illustrates a single ray of light from the point source ' $S$ ' entering the centre of the rotating cylindrical lens and striking the baseplate at a point with local plate co-ordinates of $(h, v)$. This point constitutes the centre of rotation of the line image projected onto the baseplate. The 'snapshot' shown in the figure selects that position of the line image corresponding with its incidence on one of the baseplate photocells ' $\mathrm{P}$ '. 
In practice, only two photocells, lying on two perpendicular radii from the centre of the baseplate, are needed to define a set of orthogonal co-ordinate axes, which are here labelled $\mathrm{H}-\mathrm{H}$ and $\mathrm{V}-\mathrm{V}$.

At the instant that the line image reaches the optical centre of one of the photocells, an instantaneous reading is taken of the angle encoder disk, directly linked to the rotation of the cylindrical lens. This reading together with either the preceding or succeeding latched angle reading is used by the instrument microprocessor to generate the horizontal and vertical co-ordinates of the direction vector for the target.

It is clear from Fig. 6 that the angular separation of two successive line image intercepts on a photocell will either be less than $180^{\circ}$ or greater than $180^{\circ}$ depending on whether the $\mathrm{H}-\mathrm{H}$ or V-V photocell is struck first. The order is identified in the microprocessor by the size of this angle.

The process of converting the angular separation of photocell intercepts on the baseplate into components of the target direction vector is illustrated below with the aid of Figs. 7 and 8 .

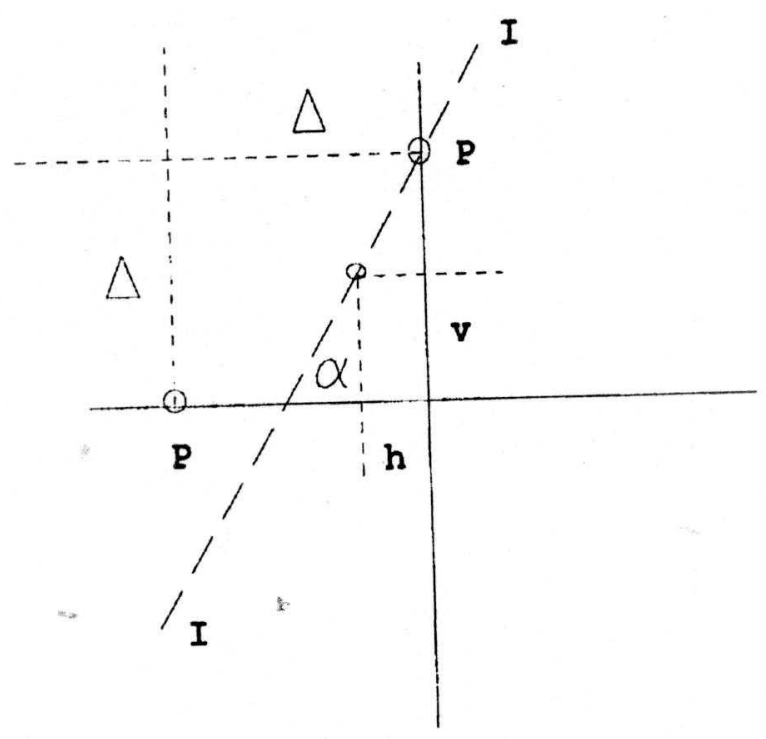

Fig. 7

In Fig. 7 the line image is shown incident on the photocell which represents the nominal 'vertical' axis of the instrument. At this instant, the angle that the line image makes with the instrument 'horizontal' axis is depicted by $\alpha$, and as before, the baseplate co-ordinates of the line image rotation point are given as $(h, v)$.
Irrespective of the inclination of the direct line to the target from the instrument and the instrument axis, the line image produced is always parallel with the cylindrical lens that produces it. Further, the rotation of the cylindrical lens is constantly monitored by the integral electronic angle encoder disk. Thus if the instrument encoder uses as its zero datum the position of the lens when parallel to the $\mathrm{H}-\mathrm{H}$ axis on the baseplate, then for the situation shown in Fig. 7, the instantaneous encoder reading for the rotating lens will be $\pi-\alpha$.

It is further assumed in the figure that each photocell is separated from the baseplate origin of co-ordinates along its respective axis by $\Delta$. The geometry of the disposition of photocells on the baseplate and setting of the encoder scale zero to correspond to the lens being parallel to the $\mathrm{H}-\mathrm{H}$ axis, are determined during initial instrument calibration.

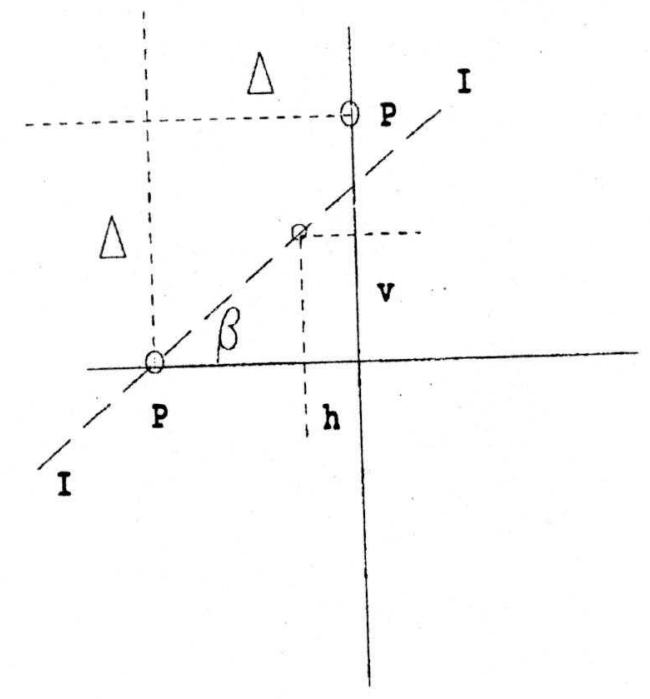

Fig. 8

Figure 8 shows the second stage of the measurement where the photocell on the $\mathrm{H}-\mathrm{H}$ axis is subsequently struck by the rotating line image. The instantaneous encoder reading that corresponds to this situation is given as $\pi-\beta$.

If the principal focal length of the cylindrical lens is $F$, then the azimuth angle of the luminous source at the theodolite (HA) is given by:

TAN $($ HA $)=h / F$

And the corresponding elevation angle (VA) by: 
TAN $($ VA $)=v / F$

Whereas from Figs. 7 and 8 ;

$\operatorname{TAN}(\alpha)=(\Delta-v) / h$

$\operatorname{TAN}(\beta)=v /(\Delta-h)$

From (3) and (4) ;

$h=(\Delta \cdot \operatorname{TAN}(\beta)) /(\operatorname{TAN}(\beta)-\operatorname{TAN}(\alpha))$

$v=\Delta-\mathbf{h} \cdot \operatorname{TAN}(\alpha)$

Direct substitution of these values into equations (1) and (2) produces the horizontal and vertical components of the full direction vector to ' $\mathrm{S}$ '.

Prototype instruments based on the foregoing principle have been constructed in IMRU and it is found that by altering the focal length of the rotary cylindrical lens used, the angular field of view of the instrument can be changed from a $10^{\circ}$ cone to one of $90^{\circ}$ without significant image distortion. The precision (repeatability) of a series of direction measures made with a prototype instrument to a LED target modulated at $10 \mathrm{MHz}$ and at 100 metres range was $+1-4$ arc seconds.

In Fig. 9 below is shown a schematic of this type of instrument, here called Autofix, performing a guidance function for a paving machine.

AUTO GUIDED ROAD PAVER

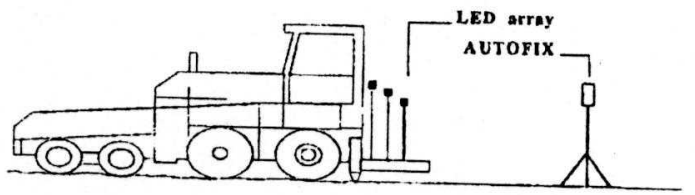

Fig. 9

The target array mounted on the paver comprises three or more modulated LEDs or semiconductor lasers directed towards the rear of the machine. The Autofix instrument occupies a known location on the work site and is set in a known direction towards the rear of the paver. The automatic direction measures made to the targets of the array at a rate of up to $30 \mathrm{~Hz}$ allow a spatial resection to be performed which generates $X, Y$ and $Z$ co-ordinates for each of the targets in the array. This enables the 3-D fixation of any known reference point on the paver as well as the three attitude co-ordinates of the paver (roll, pitch and yaw) in the co-ordinate reference system of the work-site.

As described, the new theodolite requires but a single rotating image in order to perform a direction measure to a single target. If multiple line images are generated simultaneously, then ambiguity will result. By imposing a direct high frequency modulation to the target diodes, the instrument is able to reject the effects of other ambient light sources, but this alone does not allow detection of simultaneously emitting targets. Accordingly, two techniques have been developed successfully to address this problem.

The first technique allows simultaneous emissions of the targets in the array but each emits at a different optical wavelength, say $\lambda_{1}, \lambda_{2}$ and $\lambda_{3}$. The baseplate of the instrument is then provided with three right-angle pairs of photocells, each pair being prefixed by an appropriate narrow-band optical filter which 'sees' only its intended wavelength.

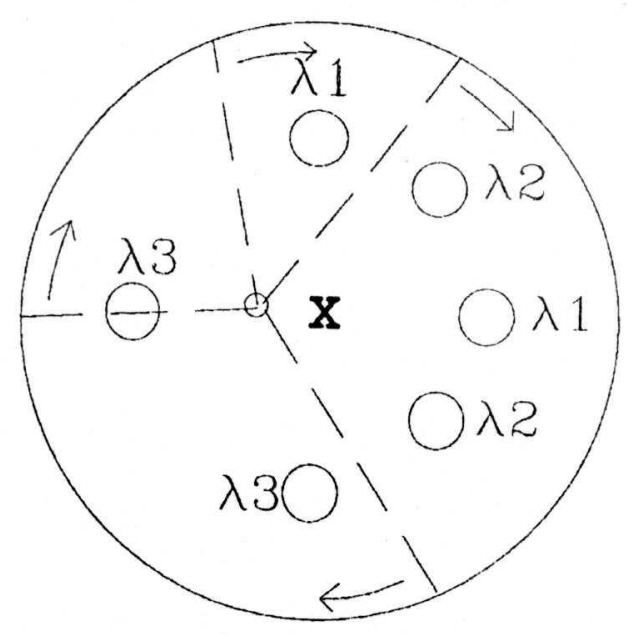

Fig. 10

In the design of the instrument, each pair of photocells is located in azimuth in relation to the internal rectangular axes of the instrument. By this means their individual measures may be correlated so that each effectively measures from the same internal axes. 
A second, and much simpler, method is merely to poll the power supply to the targets in the array. For example, if three targets are used on the paver machine in a typical pattern, one on the left, one on the right and a third near the centre of the array but displaced forward of the line joining the other two, then the following regime may be used. The leftmost target is energised by a modulated power source for 0.2 second; this is followed by energising of the middle target for 0.2 second, and likewise repeated for the rightmost target. Illumination of the targets continues in this fashion indefinitely.

The Autofix theodolite will thus measure the direction to a single target of the array every 0.2 second and, since the order of the targets in azimuth is known, the measurements data may be processed without ambiguity as if they had been obtained simultaneously.

In practice, the use of an Autofix type of theodolite is considerably enhanced if it were to be mounted on a standard electronic theodolite of which only the horizontal angle scale is used. Once the alignment, or extent of the systematic misalignment, between the main theodolite axis and that of the mounted Autofix instrument is known, the Autofix azimuth readings constitute a 'vernier' angle scale for the main horizontal circle scale of the electronic theodolite. The total vertical angle subtended by the targets in the field of view may be measured directly with Autofix.

Such a combination would allow simple and rapid data capture by Autofix, as already described, and corresponding to its full rate of rotation. Much more time would be available for reading of the electronic theodolite horizontal circle since this would be constant during the measurements. Further, the mounting of Autofix on a conventional electronic theodolite is a purely mechanical process and does not involve any electrical interfacing between the two instruments.

The main advantage of using such a combination for machine guidance purposes is that simply by using a vertically held pogo-stick carrying a single target LED on three separate site survey control points, the $\mathrm{X}, \mathrm{Y}$ and $\mathrm{Z}$ site co-ordinates for Autofix may readily be found by plane resection together with its site azimuth reference. This would allow an efficient and fairly speedy set-up for the instrument on site and ensure that the 6-D co-ordinates measured for the site machine were consistent directly with the site survey control.

\section{BIBLIOGRAPHY}

[1] Gorham B. J.

Measurement of Spatial Position using

Shaped Laser beams.

Land \& Minerals Surveying Journal:

Research, Vol. 6 March 1988

[2] Gorham B. J. Automatic Calibration of Production Robots from a single Laser source. Land \& Minerals Surveying Journal: Research, Vol. 7 January 1989

[3] Gorham B. J.

Laser Guidance System for Tunnelling Machines.

Land \& Minerals Surveying Journal:

Research, Vol. 7 September 1989

[4] Gorham B. J.

The LASERGUIDE System of Automatic

Machine Guidance.

Proc. $11^{\text {th }}$ ISARC, Brighton, UK. May 1994

[5] Gorham B. J.

A New Technique of Position Fixing for Site Vehicles.

Proc. $13^{\text {th }}$ ISARC, Tokyo, Japan. June 1996

* The instrument described in this paper is the subject of a current UK patent application. 